\title{
A comparative study of two blind FIR equalizers
}

\author{
S. Chen, ${ }^{*}$ T.B. Cook, and L.C. Anderson \\ Department of Electronics and Computer Science, University of Southampton, Highfield, \\ Southampton SO17 1BJ, UK
}

\begin{abstract}
The paper investigates blind finite-impulse-response (FIR) equalization schemes for quadrature amplitude modulation signalling. We compare a bootstrap maximum a posteriori probability (MAP) equalizer with a recently introduced concurrent constant modulus algorithm (CMA) and decision directed (DD) equalizer (CMA+DD). Both equalizers are known to outperform the CMA considerably in the situations where carrier recovery is performed and the signal constellation is known. The concurrent CMA+DD equalizer has a complexity that is slightly more than twice of the CMA, and the bootstrap MAP equalizer has computational requirements that are only slightly more complex than the CMA. Simulation results indicate that the bootstrap MAP blind FIR equalizer has a faster convergence rate and better steady-state performance than the concurrent CMA+DD blind FIR equalizer, but tuning of the former is more complicated than the latter.
\end{abstract}

(c) 2003 Elsevier Inc. All rights reserved.

Keywords: Blind equalization; Finite-impulse-response filter; Quadrature amplitude modulation; Constant modulus algorithm; Decision-directed adaptation; Maximum a posteriori probability

\section{Introduction}

Blind equalization improves system bandwidth efficiency by avoiding the use of a training sequence. Furthermore, for multi-point communication systems, training is infeasible and blind equalizer provides a practical means for combating the detrimental effects of channel intersymbol interference (ISI) in such systems. For communication systems employing high bandwidth-efficiency quadrature amplitude modulation (QAM) signalling, the constant modulus algorithm (CMA) based finite-impulse-response (FIR) equalizer is by far the most popular blind equalization scheme [1-4]. It has very simple computational

\footnotetext{
* Corresponding author.

E-mail address: sqc@ecs.soton.ac.uk (S. Chen).
} 
requirements and readily meets the real-time computational constraint. The CMA is also very robust to imperfect carrier recovery. A particular problem of the CMA, however, is that it only achieves a moderate level of mean square error (MSE) after convergence, which may not be sufficiently low for the system to obtain adequate performance. A possible solution is to switch to a decision directed (DD) adaptation which should be able to minimize the residual CMA steady state MSE [5]. However, as pointed out in [6], in order for such a transfer to be successful, the CMA steady state MSE should be sufficiently low. In practice, such a low level of MSE may not always be achievable by the CMA.

De Castro et al. [6] have suggested an interesting solution to this problem. Rather than switching to a DD adaptation after the CMA has converged, they have proposed to operate a DD equalizer concurrently with a CMA equalizer. The weight adaptation of the DD equalizer follows that of the CMA equalizer, and the DD adjustment only takes place if the CMA has achieved a successful adjustment with high probability. At a small cost of doubling complexity to that of the very simple CMA, this concurrent CMA+DD equalizer is reported to obtain a dramatical improvement in equalization performance over the CMA [6]. Many blind FIR equalizers have been reported before, which can commonly be referred to as Bussgang algorithms (e.g., [7-12]). A Bussgang-type blind equalizer has an FIR filter structure and adjusts the filter coefficients by optimizing a non-convex criterion function using stochastic gradient. The CMA is obviously a Bussgang-type blind equalizer. In the lights of the results reported in [6], we revisit a Bussgang-type blind FIR equalizer called the bootstrap maximum a posteriori probability (MAP) equalizer $[11,12]$.

The bootstrap MAP equalizer was originally derived in [13] for 4-QAM constellation and extended to $M$-QAM $(M>4)$ communication channels in [11,12]. The basic idea is to maximize the a posteriori probability density function (PDF) of the equalizer output subject to the equalizer weights. To accomplish a fast and reliable convergence and to keep the complexity to a minimum, a multi-stage procedure is adopted. At the first stage, a 4-cluster PDF model is adopted as though the data constellation is an equivalent 4-QAM one. The aim of this stage is to classify equalizer outputs correctly into one of the four quadrants in the complex plane with high probability. At the second stage, a 16-cluster PDF model is used and it is divided into 4 sub-sets, one for each quadrant. If the equalizer output appears in a particular quadrant, the corresponding 4-cluster sub-model is used to adapt the equalizer weights. After the stage two, the complex plane is divided into 16 square regions, each containing a 4-cluster sub-model. The procedure is continuing until after the $L$ th stage, where $L$ is given by $2^{L}=\sqrt{M}$, the correct data constellation is restored.

In this study, we compare the bootstrap MAP equalizer with the concurrent CMA+DD equalizer, with the standard CMA acting as a benchmark in terms of complexity and performance. Note that we assume carrier lock and constellation registration. Obviously, the CMA does not actually need these assumptions. However, in normal communication systems, the signal constellation is known to receiver and, typically, carrier recovery is performed. The bootstrap MAP equalizer requires similar numbers of multiplications and additions as the CMA, with an additional need of evaluating 4 exponential function values. Even taking into account this additional requirement, the complexity of the bootstrap MAP equalizer is very simple and is less than that of the concurrent CMA+DD equalizer. As the bootstrap MAP equalizer requires $L$-stage switching and each stage has a set of different algorithm parameters, the tuning of the bootstrap MAP equalizer is more complicated than 
the concurrent CMA+DD equalizer. Simulation confirms that both the bootstrap MAP and concurrent CMA+DD equalizers outperform the CMA considerably, and the results show that the bootstrap MAP equalizer achieves faster convergence and better steady-state performance than the concurrent CMA+DD equalizer.

\section{Blind equalization}

Blind equalization with a fractionally-spaced equalizer (FSE) is investigated. For simplicity, we will consider the $T_{S} / 2$-spaced FSE, where $T_{S}$ denotes the symbol period. The baseband discrete-time model of communication system with a $T_{S} / 2$-spaced FSE is depicted in Fig. 1 (see [14]). For notational convenience, the index $k$ is reserved for $T_{s}$-spaced quantities and index $n$ for $T_{S} / 2$-spaced quantities throughout the discussion. The transmitted $T_{s}$-spaced complex symbol sequence $s(k)=s_{R}(k)+j s_{I}(k)$ is assumed to be independently identically distributed (i.i.d.) and the symbol constellation is $M$-QAM with the set of all the symbol points defined by

$$
\mathcal{S}=\left\{s_{i l}=(2 i-Q-1)+j(2 l-Q-1), 1 \leqslant i, l \leqslant Q\right\},
$$

where $Q=\sqrt{M}=2^{L}$, and $L$ is an integer. The received $T_{S} / 2$-spaced signal sample is

$$
\bar{r}(n)=\sum_{i=0}^{2 N_{c}-1} \bar{a}_{i} \bar{s}(n-i)+\bar{e}(n),
$$

where the $T_{S} / 2$-spaced sequence $\{\bar{s}(n)\}$ is a zero-filled version of the transmitted symbol sequence $\{s(k)\}$ defined by

$$
\bar{s}(n)= \begin{cases}s(n / 2), & \text { for even } n, \\ 0, & \text { for odd } n,\end{cases}
$$

the channel is specified by the $T_{S} / 2$-spaced complex-valued channel impulse response (CIR) given by

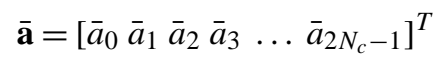

with $N_{c}$ corresponding to the $T_{s}$-spaced CIR length, and the $T_{s} / 2$-spaced sample $\bar{e}(n)=$ $\bar{e}_{R}(n)+j \bar{e}_{I}(n)$ is an i.i.d. complex Gaussian white noise with $\mathrm{E}\left[\bar{e}_{R}^{2}(n)\right]=\mathrm{E}\left[\bar{e}_{I}^{2}(n)\right]=\sigma_{e}^{2}$, and $\mathrm{E}[\cdot]$ denotes the expectation operator.

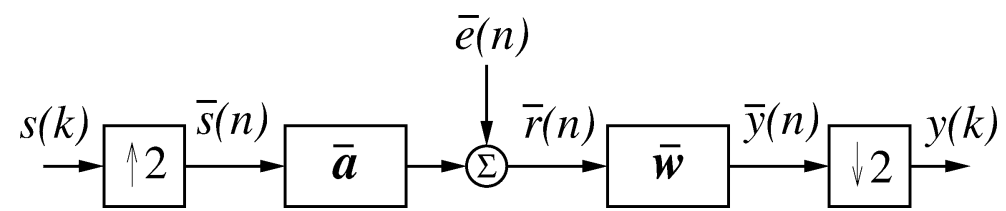

Fig. 1. Multirate baseband model of communication system with $T_{S} / 2$-spaced equalizer, where $T_{S}$ denotes symbol period, index $k$ indicates $T_{S}$-spaced quantities, and index $n$ indicates $T_{S} / 2$-spaced quantities. 
To remove the channel distortion, a $T_{S} / 2$-spaced equalizer is employed, which has an FIR structure defined by

$$
\bar{y}(n)=\sum_{i=0}^{2 m-1} \bar{w}_{i} \bar{r}(n-i)=\overline{\mathbf{w}}^{T} \overline{\mathbf{r}}(n),
$$

where $2 m$ is the order or length of the $T_{S} / 2$-spaced equalizer,

$$
\overline{\mathbf{w}}=\left[\begin{array}{llll}
\bar{w}_{0} & \bar{w}_{1} & \ldots & \bar{w}_{2 m-1}
\end{array}\right]^{T}
$$

is the equalizer complex-valued weight vector, and

$$
\overline{\mathbf{r}}(n)=[\bar{r}(n) \bar{r}(n-1) \ldots \bar{r}(n-2 m+1)]^{T}
$$

is the equalizer input vector. To deal with non-minimum phase channels, the equalizer should have a decision delay approximately to $m$. Before blind adaptation, the equalizer weights are initialized to $\bar{w}_{i}=1+j 0$ for $i=m-1$ and $m$, and $\bar{w}_{i}=0+j 0$ for all the other values of $i$. The FSE output $\bar{y}(n)$ is decimated by a factor of 2 to create the $T_{s}$-spaced output $y(k)$.

It can easily be shown [14] that the system model of Fig. 1 is equivalent to the model depicted in Fig. 2 by defining

$$
\begin{aligned}
& \overline{\mathbf{a}}^{\mathrm{e}}=\left[\begin{array}{llll}
\bar{a}_{0} & \bar{a}_{2} & \ldots & \bar{a}_{2 N_{c}-2}
\end{array}\right]^{T}, \\
& \overline{\mathbf{w}}^{\mathrm{e}}=\left[\begin{array}{llll}
\bar{w}_{0} & \bar{w}_{2} & \ldots & \bar{w}_{2 m-2}
\end{array}\right]^{T},
\end{aligned}
$$

and

$$
\begin{aligned}
e^{\mathrm{e}}(k) & =\bar{e}(2 n), & & e^{\mathrm{o}}(k)=\bar{e}(2 n+1), \\
r^{\mathrm{e}}(k) & =\bar{r}(2 n), & r^{\mathrm{o}}(k) & =\bar{r}(2 n+1) .
\end{aligned}
$$

Further define

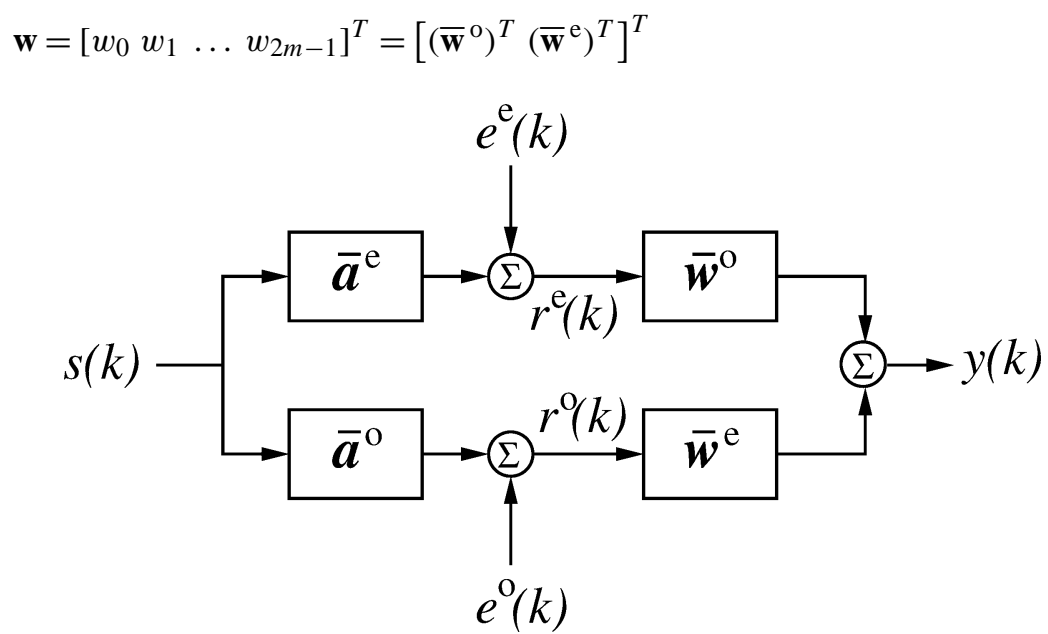

Fig. 2. Multichannel model of communication system with $T_{S} / 2$-spaced equalizer, where $T_{S}$ denotes symbol period, and index $k$ indicates $T_{S}$-spaced quantities. 
and

$$
\mathbf{r}(k)=[r(k) r(k-1) \ldots r(k-2 m+1)]^{T}=\left[\left(\mathbf{r}^{\mathrm{e}}(k)\right)^{T}\left(\mathbf{r}^{\mathrm{o}}(k)\right)^{T}\right]^{T}
$$

with $\mathbf{r}^{\mathrm{e}}(k)=\left[r^{\mathrm{e}}(k) r^{\mathrm{e}}(k-1) \ldots r^{\mathrm{e}}(k-m+1)\right]^{T}$ and $\mathbf{r}^{\mathrm{o}}(k)=\left[r^{\mathrm{o}}(k) r^{\mathrm{o}}(k-1) \ldots r^{\mathrm{o}}(k-\right.$ $m+1)]^{T}$. Then the $T_{s}$-spaced equalizer output $y(k)$ is given by

$$
y(k)=\sum_{i=0}^{2 m-1} w_{i} r(k-i)=\mathbf{w}^{T} \mathbf{r}(k) .
$$

The equalizer model (12) forms the basis for the discussion of the three blind adaptive algorithms in the following subsections.

\subsection{Constant modulus algorithm}

The CMA adjusts the equalizer weights by minimizing the non-convex cost function

$$
\bar{J}_{\mathrm{CMA}}(\mathbf{w})=\mathrm{E}\left[\left(|y(k)|^{2}-\Delta_{2}\right)^{2}\right]
$$

using a stochastic gradient algorithm, where $\Delta_{2}$ is a real positive constant defined by

$$
\Delta_{2}=\mathrm{E}\left[|s(k)|^{4}\right] / \mathrm{E}\left[|s(k)|^{2}\right] .
$$

At $T_{s}$-spaced sample $k$, given $y(k)=\mathbf{w}^{T}(k) \mathbf{r}(k)$, the CMA adapts $\mathbf{w}$ according to [1,2]

$$
\left\{\begin{array}{l}
\epsilon(k)=y(k)\left(\Delta_{2}-|y(k)|^{2}\right), \\
\mathbf{w}(k+1)=\mathbf{w}(k)+\mu \epsilon(k) \mathbf{r}^{*}(k),
\end{array}\right.
$$

where $\mu$ is a small positive adaptive gain and $\mathbf{r}^{*}(k)$ is the complex conjugate of $\mathbf{r}(k)$.

The CMA is by far the most popular blind equalizer for high-order QAM signal constellation. It has a very simple computational complexity, as summarized in Table 1. Although $M$-QAM symbols do not fall on the circle of radius $\sqrt{\Delta_{2}}$, it is known that the cost function $\bar{J}_{\mathrm{CMA}}(\mathbf{w})$ is minimized at the equalizer weight solution which restores the signal constellation. Under certain conditions, the CMA converges to this solution subject to a possible phase shift. Let $\mathbf{w}_{\text {opt }}$ be the solution of the adaptive equalizer based on the cost function (13) that yields the correct signal constellation. All the weight vectors

$$
\mathbf{w}_{s}=\exp (j \phi) \mathbf{w}_{\mathrm{opt}}, \quad 0 \leqslant \phi<2 \pi,
$$

produces the same cost as $\bar{J}_{\mathrm{CMA}}\left(\mathbf{w}_{\mathrm{opt}}\right)$. In practice, the adaptive equalizer may converge to any of the solutions defined in (16). This undesired phase shift cannot be resolved by the CMA and must be eliminated by other means. Typically, a very small adaptive gain $\mu$ has to be used to ensure convergence.

Table 1

Comparison of computational complexity per weight update. The equalizer order is $2 m$

\begin{tabular}{lccc}
\hline Equalizer & Multiplications & Additions & Exp $(\cdot)$ evaluations \\
\hline CMA & $8 \times(2 m)+6$ & $8 \times(2 m)$ & - \\
CMA+DD & $16 \times(2 m)+8$ & $20 \times(2 m)$ & - \\
MAP & $8 \times(2 m)+23$ & $8 \times(2 m)+19$ & 4 \\
\hline
\end{tabular}




\subsection{Concurrent CMA and decision directed equalizer}

De Castro et al. [6] proposed a blind equalization scheme that consists of a CMA equalizer and a DD equalizer operating concurrently. Specifically, let

$$
\mathbf{w}=\mathbf{w}_{c}+\mathbf{w}_{d},
$$

where $\mathbf{w}_{c}$ is the weight vector of the CMA equalizer which is designed to minimize the CMA cost function $\bar{J}_{\mathrm{CMA}}\left(\mathbf{w}_{c}\right)$ and $\mathbf{w}_{d}$ is the weight vector of the DD equalizer which is designed to minimize the decision based MSE

$$
\bar{J}_{\mathrm{DD}}\left(\mathbf{w}_{d}\right)=\frac{1}{2} \mathrm{E}\left[|\mathcal{Q}[y(k)]-y(k)|^{2}\right]
$$

with $\mathcal{Q}[y(k)]$ denoting the quantized equalizer output defined by

$$
\mathcal{Q}[y(k)]=\arg \min _{s_{i l} \in \mathcal{S}}\left|y(k)-s_{i l}\right|^{2} .
$$

More precisely, at $k$, given $y(k)=\mathbf{w}_{c}^{T}(k) \mathbf{r}(k)+\mathbf{w}_{d}^{T}(k) \mathbf{r}(k)$, the CMA part adapts $\mathbf{w}_{c}$ according to the rule (15) by substituting $\mathbf{w}_{c}$ in the place of $\mathbf{w}$ with an adaptive gain $\mu_{c}$. The DD adaptation follows immediately after the CMA adaptation and it only takes place if the CMA adjustment is viewed to be a successful one. Let

$$
\tilde{y}(k)=\mathbf{w}_{c}^{T}(k+1) \mathbf{r}(k)+\mathbf{w}_{d}^{T}(k) \mathbf{r}(k) .
$$

Then the DD part adjusts $\mathbf{w}_{d}$ according to [6]

$$
\mathbf{w}_{d}(k+1)=\mathbf{w}_{d}(k)+\mu_{d} \delta(\mathcal{Q}[\tilde{y}(k)]-\mathcal{Q}[y(k)])(\mathcal{Q}[y(k)]-y(k)) \mathbf{r}^{*}(k),
$$

where $\mu_{d}$ is the adaptive gain of the DD equalizer and the indicator function

$$
\delta(x)= \begin{cases}1, & x=0+j 0, \\ 0, & x \neq 0+j 0 .\end{cases}
$$

It can be seen that $\mathbf{w}_{d}$ is updated only if the equalizer hard decisions before and after the CMA adaptation are the same.

The complexity of this CMA+DD blind equalizer, summarized in Table 1, is obviously linear in the equalizer order $2 m$. Let $\mathbf{w}_{d \text { opt }}$ be the solution of the DD equalizer based on the cost function (18) that yields the correct signal constellation. The weight vectors

$$
\mathbf{w}_{d s}=\exp (j \phi) \mathbf{w}_{d \mathrm{opt}}, \quad \phi=0, \frac{\pi}{2}, \pi, \frac{3 \pi}{2},
$$

produces the same cost as $\bar{J}_{\mathrm{DD}}\left(\mathbf{w}_{d \text { opt }}\right)$. As with any blind equalization scheme, this ambiguity needs to be resolved by other means. However, the DD adaptation does not suffer from a serious phase shift problem. Note that the CMA is very robust and is capable of opening "initially closed eye." The decision-directed adaptation, when is safe to perform, has a much faster convergence speed and is capable of lowering the steady state MSE, compared with the CMA. Obviously, this CMA+DD blind equalizer combines the advantages of both the CMA and decision-directed adaptation. The adaptive gain $\mu_{d}$ for the DD equalizer can often be chosen much larger than $\mu_{c}$ used for the CMA. 


\subsection{Bootstrap MAP equalizer}

After the equalization is accomplished, the equalizer output can approximately be expressed in two terms

$$
y(k) \approx x(k)+v(k),
$$

where $x(k)=s\left(k-k_{d}\right), k_{d}$ is an integer, and $v(k)=v_{R}(k)+j v_{I}(k)$ is approximately a Gaussian white noise. Thus, if the equalizer weights have correctly been chosen, the equalizer output can be modelled approximately by $M$ Gaussian clusters. The cluster means are

$$
y_{i l}=s_{i l}, \quad 1 \leqslant i, l \leqslant Q,
$$

and all the clusters have an approximate covariance

$$
\left[\begin{array}{cc}
\mathrm{E}\left[v_{R}^{2}(k)\right] & \mathrm{E}\left[v_{R}(k) v_{I}(k)\right] \\
\mathrm{E}\left[v_{I}(k) v_{R}(k)\right] & \mathrm{E}\left[v_{I}^{2}(k)\right]
\end{array}\right] \approx\left[\begin{array}{cc}
\rho & 0 \\
0 & \rho
\end{array}\right] .
$$

Under the above conditions, the a posteriori PDF of $y(k)$ is approximately

$$
p(\mathbf{w}, y(k)) \approx \sum_{q=1}^{Q} \sum_{l=1}^{Q} \frac{p_{q l}}{2 \pi \rho} \exp \left(-\frac{\left|y(k)-y_{q l}\right|^{2}}{2 \rho}\right),
$$

where $p_{q l}$ are the a priori probabilities of $y_{q l}, 1 \leqslant q, l \leqslant Q$, and they are all equal. The bootstrap MAP equalizer is designed to maximize log of the a posteriori PDF criterion

$$
\bar{J}_{\mathrm{MAP}}(\mathbf{w})=\mathrm{E}\left[J_{\mathrm{MAP}}(\mathbf{w}, y(k))\right]
$$

with

$$
J_{\mathrm{MAP}}(\mathbf{w}, y(k))=\rho \log (p(\mathbf{w}, y(k))) .
$$

At $k$, given $y(k)=\mathbf{w}^{T}(k) \mathbf{r}(k)$, the equalizer weights can be adapted according to the stochastic gradient algorithm

$$
\mathbf{w}(k+1)=\mathbf{w}(k)+\mu \frac{\partial J_{\mathrm{MAP}}(\mathbf{w}(k), y(k))}{\partial \mathbf{w}}
$$

with the adaptive gain $\mu$ and the stochastic gradient

$$
\frac{\partial J_{\mathrm{MAP}}(\mathbf{w}(k), y(k))}{\partial \mathbf{w}}=\frac{\sum_{q=1}^{Q} \sum_{l=1}^{Q} \exp \left(-\frac{\left|y(k)-y_{q}\right|^{2}}{2 \rho}\right)\left(y_{q l}-y(k)\right)}{\sum_{q=1}^{Q} \sum_{l=1}^{Q} \exp \left(-\frac{\left|y(k)-y_{q l}\right|^{2}}{2 \rho}\right)} \mathbf{r}^{*}(k) .
$$

Karaoguz and Ardalan [13] first suggested this algorithm for 4-QAM $(Q=2)$ channels. In order to speed up convergence rate and to keep the complexity to a minimum, a multi-stage implementation was proposed [11,12] for high-oder QAM signalling.

In the 16-QAM case, the equalization objective is decomposed into a two-stage process. In the first stage, a 4-cluster PDF model is adopted with the 4 cluster means being $\{ \pm 2+$ $j \pm 2\}$. The equalizer weights are adjusted using this equivalent "4-QAM" model through the gradient algorithm (30). The objective of this stage is to achieve a roughly correct classification of equalizer outputs into the 4 quadrants in the complex plane, and this task 
can easily be accomplished. At the second stage, the 16-cluster PDF model is adopted with the 16 cluster means being the correct symbol points $\left\{s_{q l}, 1 \leqslant q, l \leqslant 4\right\}$. This cluster model is divided into 4 sub-models, one for each quadrant. If the equalizer output is in a particular quadrant, the corresponding 4-cluster sub-model is used to adapt the equalizer weights via the gradient algorithm (30). The equalizer adaptation is done correctly with high probability at this stage owing to the primary clustering of the previous stage. Thus the overall equalization objective can be achieved faster and more reliably.

For the 64-QAM case, a three-stage process is adopted. This multi-stage process is upwardly extendable. In general, the task of $M$-QAM equalization, where $M=2^{2 L}$, can be achieved using the $L$-stage process. Because the sub-task of each stage can be accomplished easily and reliably, the overall convergence of the equalizer is achieved faster and more reliably. The soft-decision directed nature of this bootstrap MAP means that a much large adaptive gain can be used, which otherwise would cause the CMA to diverge. The choice of $\rho$ for each adaptation stage should ensure a proper separation of the clusters. If the value of $\rho$ is too large, a desired degree of separation among the clusters may not be achieved. On the other hand, if a too small $\rho$ is used, the algorithm attempts to impose a very tight control in the size of clusters and may fail to do so. Apart from these two extreme cases, the performance of the algorithm does not critically depend on the value of $\rho$, and there exists a wide range of values for $\rho$ at each stage of the adaptation.

It is obvious that the criterion (28) is maximized when the equalizer output produces the correctly signal constellation. Let $\mathbf{w}_{\text {opt }}$ be the solution of the adaptive equalizer based on the criterion (28) that yields the correct signal constellation. Then the weight vectors which produce the same function value as $\bar{J}_{\mathrm{MAP}}\left(\mathbf{w}_{\text {opt }}\right)$ are given by

$$
\mathbf{w}_{s}=\exp (j \phi) \mathbf{w}_{\text {opt }}, \quad \phi=0, \frac{\pi}{2}, \pi, \frac{3 \pi}{2} .
$$

It can be seen that the bootstrap MAP equalizer does not suffer from a serious phase shift problem as the CMA does. Since the equalizer weights are always adapted using a 4-cluster sub-model at each sample via the gradient algorithm (30), the complexity is always compatible to the minimum complexity of the 4-QAM case, and is only slightly more than the CMA, as can be seen from Table 1 . The $4 \exp (\cdot)$ evaluations can be implemented through look up table in practice. Because each stage of the bootstrap MAP equalizer requires a different set of algorithm parameters, the tuning of the bootstrap MAP equalizer is more complicated than the concurrent CMA+DD one.

\section{Simulation study}

The performance of the concurrent CMA+DD and bootstrap MAP blind equalizers were evaluated in a computer simulation using the standard CMA blind equalizer as a benchmark. Two performance criteria were used to assess the convergence rate of a blind equalizer. The first one was an estimated MSE at each adaptation sample based on a block of $N_{\mathrm{MSE}} T_{s}$-spaced data samples

$$
\mathrm{MSE}=\frac{1}{N_{\mathrm{MSE}}} \sum_{k=1}^{N_{\mathrm{MSE}}}|\mathcal{Q}[y(k)]-y(k)|^{2} .
$$


Note that this is the decision based MSE, not the true MSE achievable by an equalizer. The second one was the maximum distortion (MD) measure defined by

$$
\mathrm{MD}=\frac{\sum_{i=0}^{N_{f}-1}\left|f_{i}\right|-\left|f_{i_{\max }}\right|}{\left|f_{i_{\max }}\right|},
$$

where $\left\{f_{i}\right\}_{i=0}^{N_{f}-1}$ was the combined impulse response of the channel and equalizer defined by $\overline{\mathbf{w}}^{\mathrm{o}} \star \overline{\mathbf{a}}^{\mathrm{e}}+\overline{\mathbf{w}}^{\mathrm{e}} \star \overline{\mathbf{a}}^{\mathrm{o}}$ with $\star$ denoting convolution and $N_{f}=N_{c}+m-1$ being the length of the $T_{s}$-spaced combined impulse response, and

$$
f_{i_{\max }}=\max \left\{f_{i}, 0 \leqslant i \leqslant N_{f}-1\right\} .
$$

The equalizer output signal constellation after convergence was also shown using $N_{\text {test }}=$ $6000 T_{s}$-spaced testing data samples not used in adaptation.

The $T_{S} / 2$-spaced equalizer order $2 m$ should be chosen sufficiently long to ensure the capability of opening closed eye and good steady-state performance, but not too long which can cause the problems of seriously enhancing noise and slow convergence rate. The actual value of $2 m$ used in the simulation was found empirically. The values for various adaptive algorithm parameters, namely adaptive gains for the CMA, DD and MAP, and widths for different stages of the MAP equalizer, were also chosen empirically to ensure fast convergence speed and good steady-state performance.

\section{Example 1}

The CIR, listed in Table 2, was a simulated $T_{S} / 2$-spaced 6-tap channel and the data symbols were 16-QAM. The noise power was $\sigma_{e}^{2}=0.0435$, giving rise to a channel signal to noise ratio (SNR) of $20 \mathrm{~dB}$. The blind equalizer had $18 T_{S} / 2$-spaced taps, and the length of data samples for estimating the MSE at each adaptation sample was $N_{\mathrm{MSE}}=250$. A length of $2 m=18$ for the $T_{S} / 2$-spaced blind equalizer was found to be sufficient to achieve a perfect reconstruction in the noise-free case. The adaptive gain for the CMA was $\mu=0.00001$, and the two adaptive gains of the concurrent CMA+DD equalizer were set to $\mu_{c}=0.00001$ and $\mu_{d}=0.0005$. For the bootstrap MAP equalizer, $1000 T_{s}$-spaced samples were used in the first stage with $\mu=0.0005$ and $\rho=1.6$, while in the second stage the adaptive gain was $\mu=0.0005$ with the cluster width $\rho=0.6$. Notice that the adaptive gain for the CMA had to be chosen so small to avoid divergence.

Table 2

A simulated $T_{S} / 2$-spaced 6-tap channel impulse response, where $T_{S}$ denotes symbol period

\begin{tabular}{crc}
\hline Tap no. & Real & Imaginary \\
\hline 0 & -0.2 & 0.3 \\
1 & -0.5 & 0.4 \\
2 & 0.7 & -0.6 \\
3 & 0.4 & 0.3 \\
4 & 0.2 & 0.1 \\
5 & -0.1 & 0.2 \\
\hline
\end{tabular}




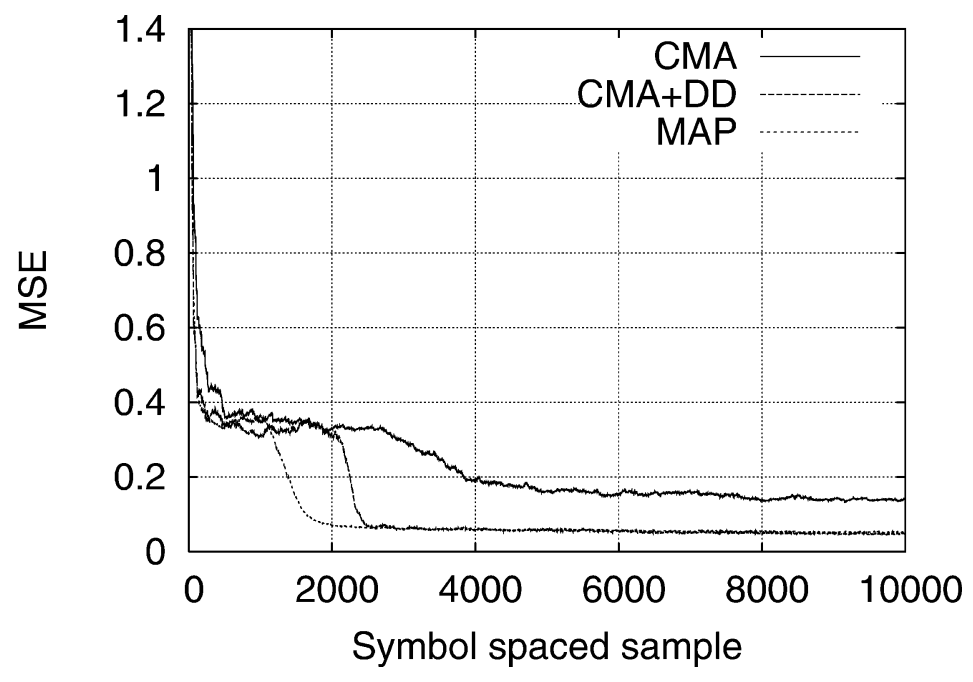

(a)

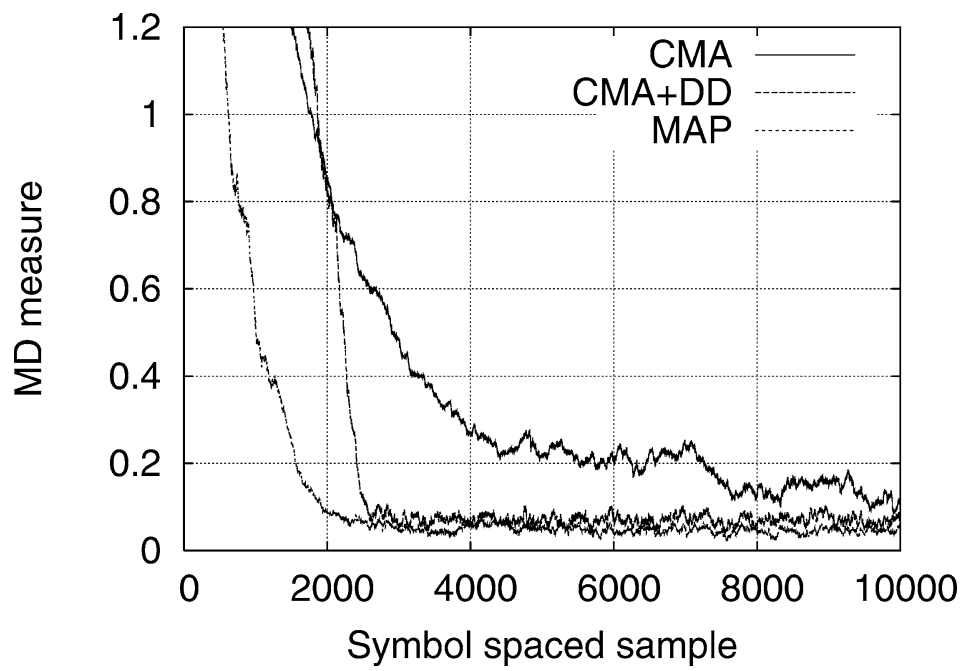

(b)

Fig. 3. Comparison of convergence performance in terms of (a) estimated MSE and (b) MD measure for Example 1.

The learning curves of the three blind equalizers, the CMA, the CMA+DD and the bootstrap MAP, are compared in Fig. 3. The equalizer output signal constellations after convergence are shown in Fig. 4. The results confirm the founding of [6] that the concurrent CMA+DD equalizer has superior performance over the pure CMA. It can also be seen that for this example the bootstrap MAP equalizer has the fastest convergence rate and the lowest MD measure among the three blind equalizers. The signal constellation of the CMA shown in Fig. 4b has an obvious phase rotation. 


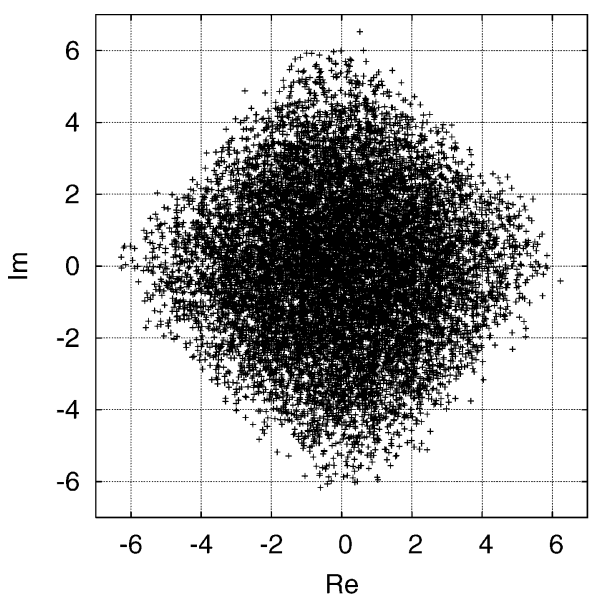

(a)

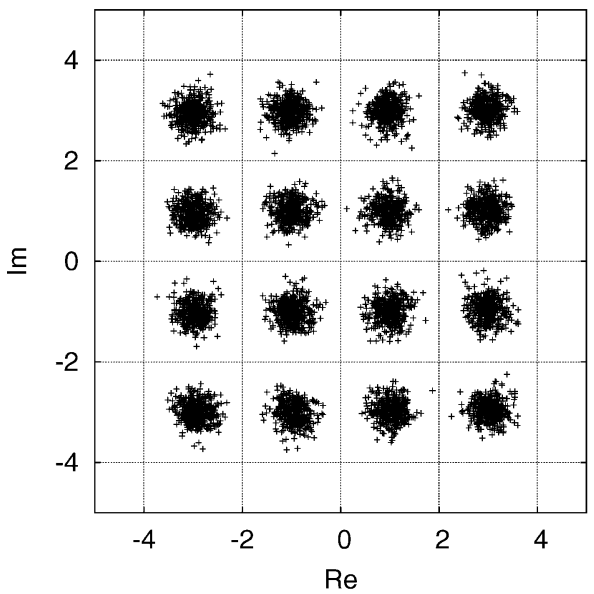

(c)

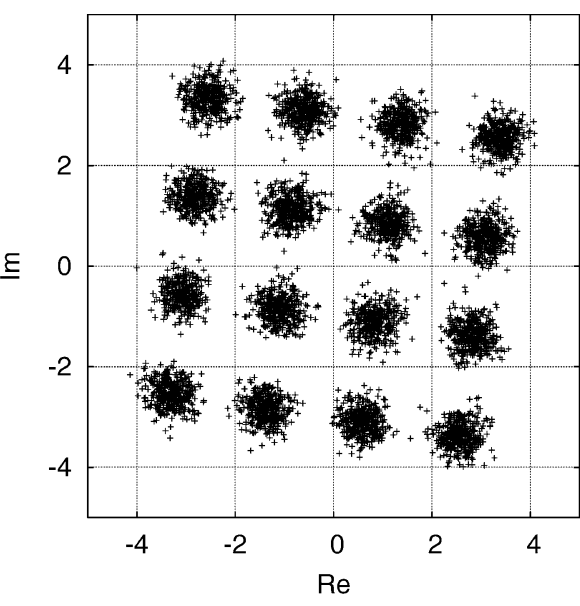

(b)

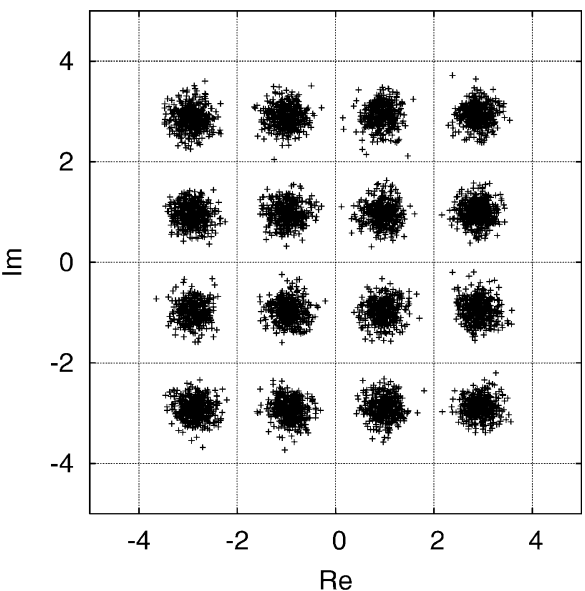

(d)

Fig. 4. Equalizer $T_{S} / 2$-spaced input (a), and equalizer $T_{S}$-spaced output signal constellations after convergence (b) the CMA, (c) the CMA+DD, and (d) bootstrap MAP for Example 1.

\section{Example 2}

The CIR was again given in Table 2 but the transmitted data symbols were 64-QAM. Given a noise power of $\sigma_{e}^{2}=0.00183$, the SNR was $40 \mathrm{~dB}$. The $T_{s} / 2$-spaced equalizer had 18 taps and the length of data samples for estimating the MSE at each adaptation was $N_{\mathrm{MSE}}=500$. The CMA had an adaptive gain $\mu=5 \times 10^{-7}\left(\mu=10^{-6}\right.$ caused divergence $)$, and the two adaptive gains of the concurrent CMA+DD equalizer were $\mu_{c}=5 \times 10^{-7}$ and 


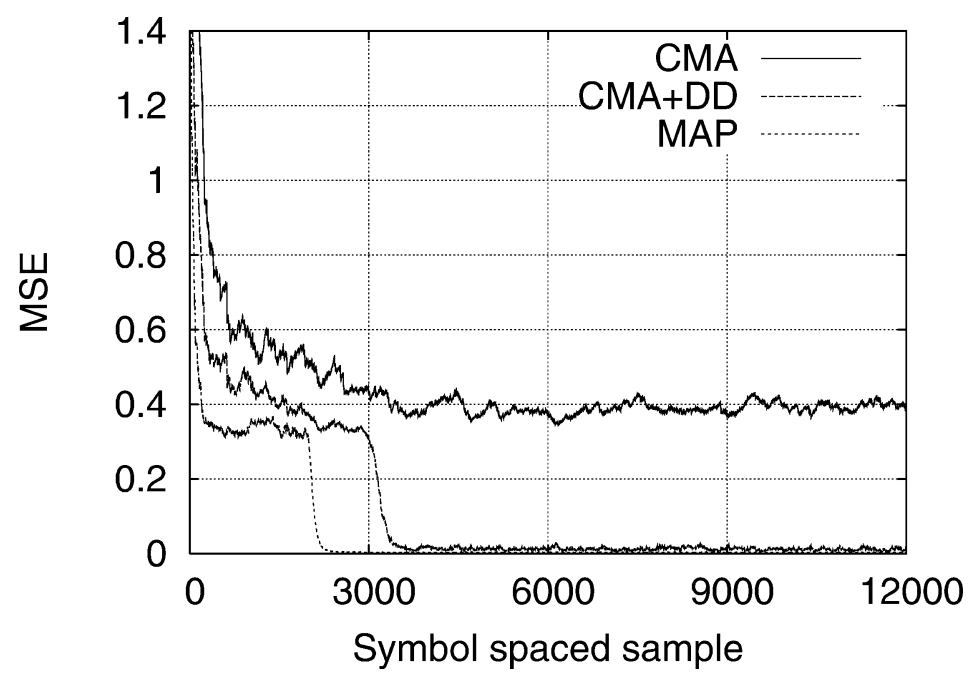

(a)

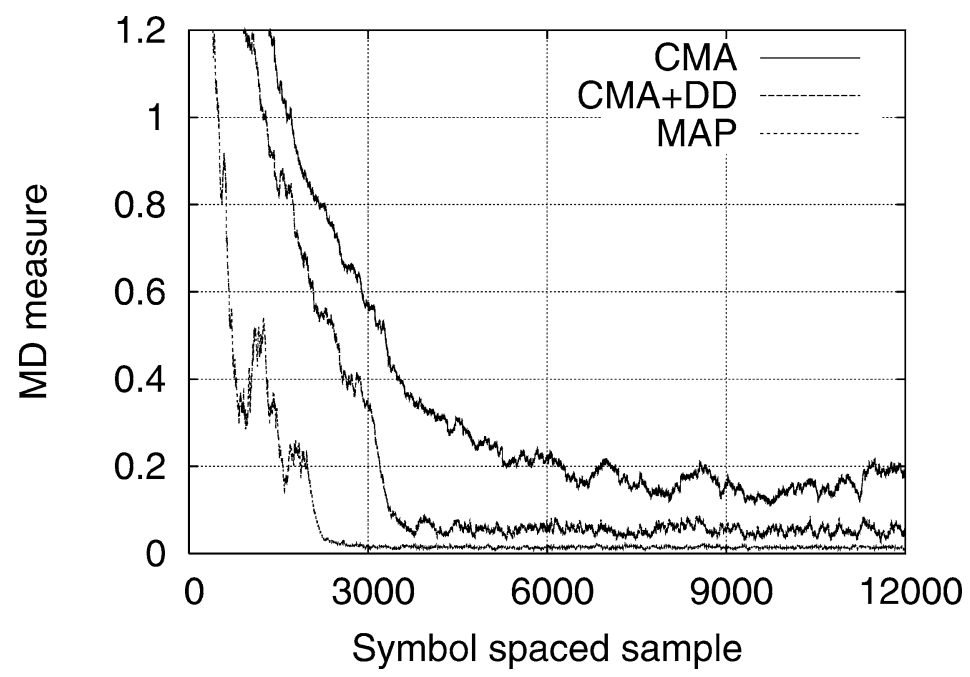

(b)

Fig. 5. Comparison of convergence performance in terms of (a) estimated MSE and (b) MD measure for Example 2 .

$\mu_{d}=0.0001$. For the first stage of the bootstrap MAP equalizer, $1000 T_{s}$-spaced samples were used with $\mu=0.0002$ and $\rho=8.0$; for the second stage 1000 samples were used with $\mu=0.0002$ and $\rho=1.6$; in the final third stage, the adaptive gain was $\mu=0.0002$ with the cluster width $\rho=0.6$.

The convergence performance of the three blind equalizers, in terms of the estimated MSE and MD measure, are depicted in Figs. 5a and 5b, respectively. It can clearly be seen that both the concurrent CMA+DD and bootstrap MAP equalizers have much better 


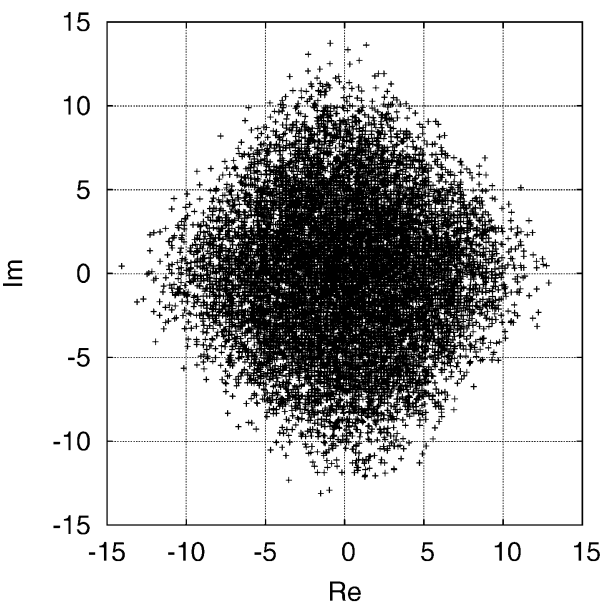

(a)

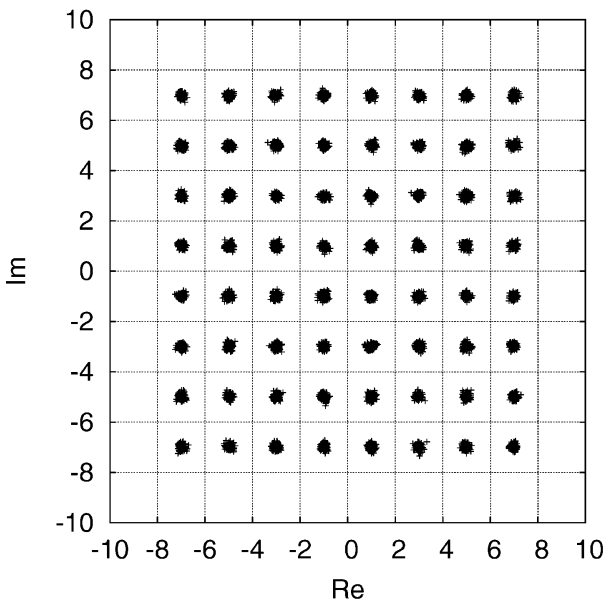

(c)

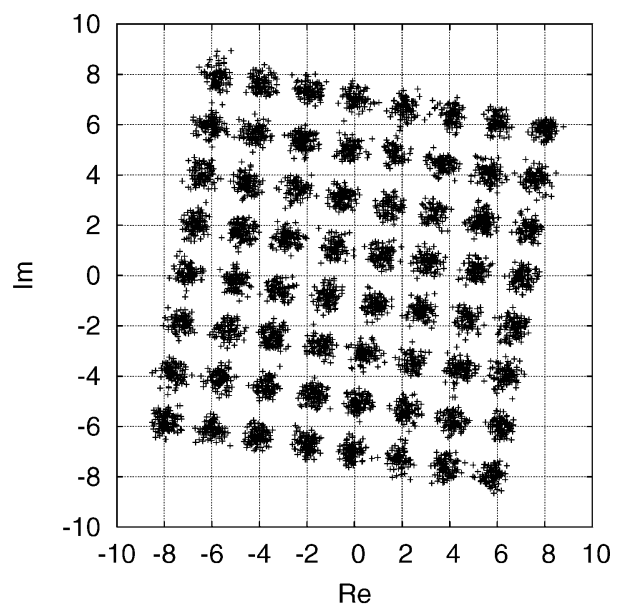

(b)

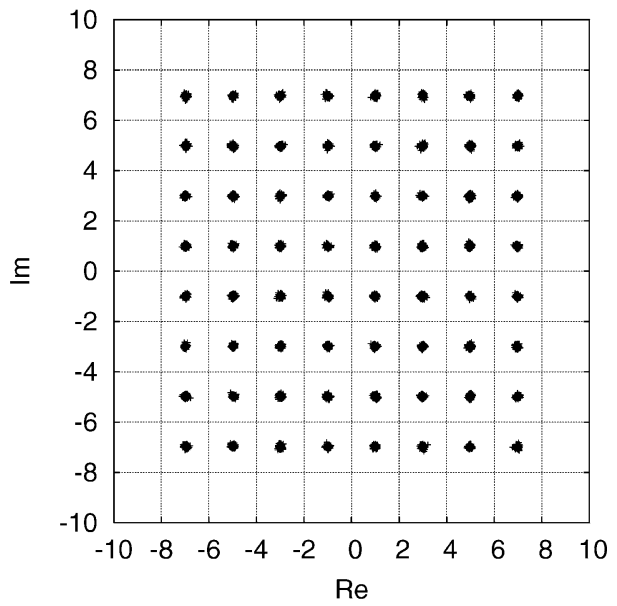

(d)

Fig. 6. Equalizer $T_{S} / 2$-spaced input (a), and equalizer $T_{S}$-spaced output signal constellations after convergence (b) the CMA, (c) the CMA+DD, and (d) bootstrap MAP for Example 2.

equalization performance over the CMA. The three equalizer output signal constellations are shown in Fig. 6, where a phase rotation of the CMA signal constellation is evident in Fig. 6b. The signal constellation of the bootstrap MAP equalizer depicted in Fig. $6 \mathrm{~d}$ has the best quality among the three blind equalizers. For this example the bootstrap MAP equalizer has a faster convergence speed and better steady-state performance than the concurrent CMA+DD equalizer. 


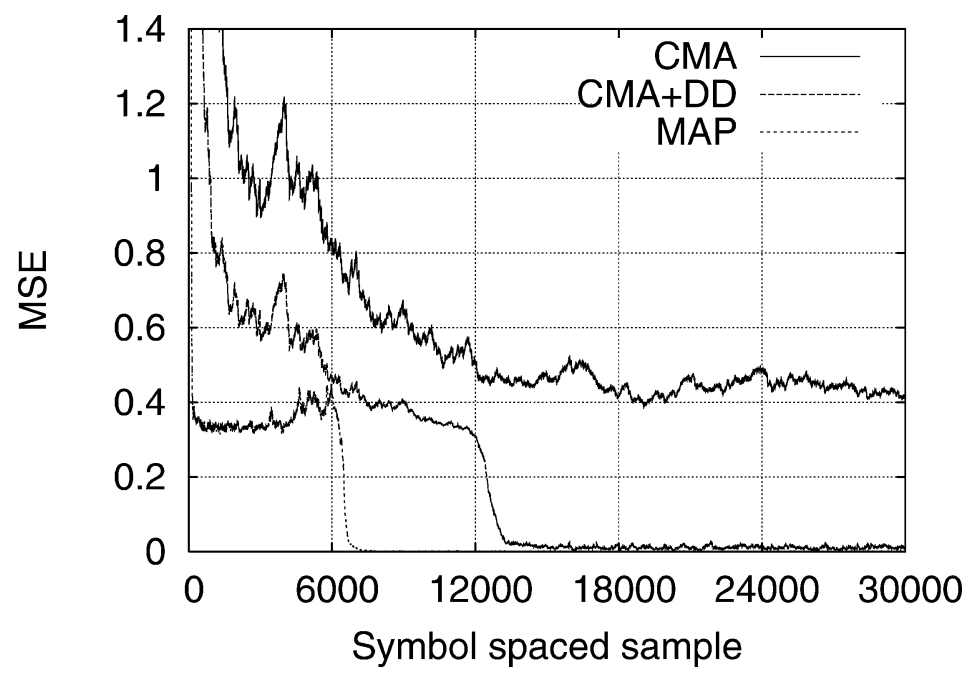

(a)

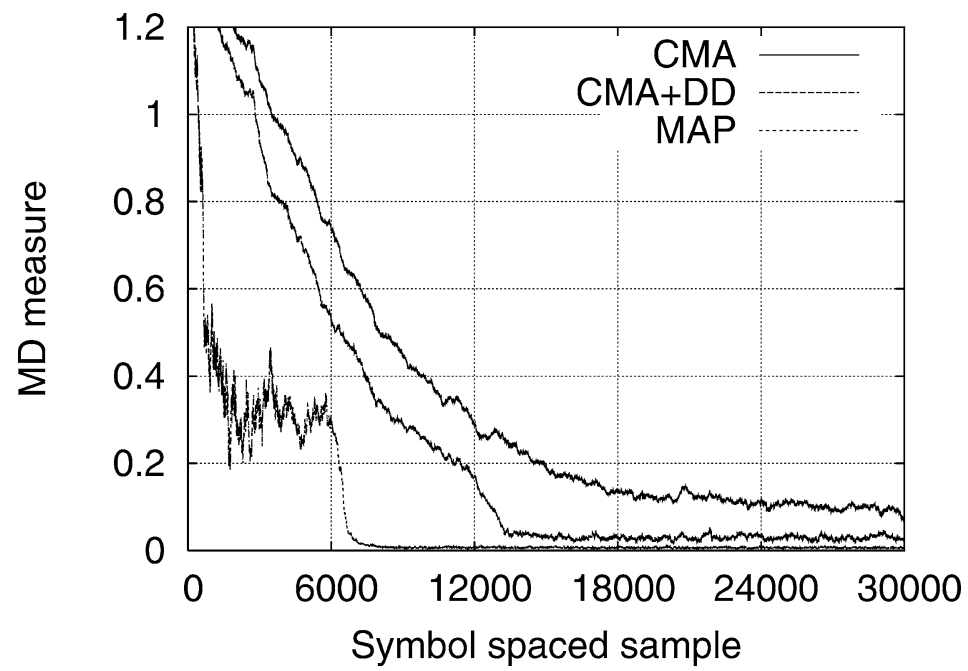

(b)

Fig. 7. Comparison of convergence performance in terms of (a) estimated MSE and (b) MD measure for Example 3 .

\section{Example 3}

In this example, 256-QAM data symbols were transmitted through the channel whose CIR is given in Table 2. The noise power was set to $\sigma_{e}^{2}=7.39 \times 10^{-5}$, corresponding to a SNR of $60 \mathrm{~dB}$. The $T_{S} / 2$-spaced equalizer had 18 taps and the length of the data block for estimating the MSE at each adaptation was $N_{\mathrm{MSE}}=1000$. The adaptive gain for the CMA had to be set to $\mu=10^{-8}$ to avoid divergence. The two adaptive gains of the concurrent 


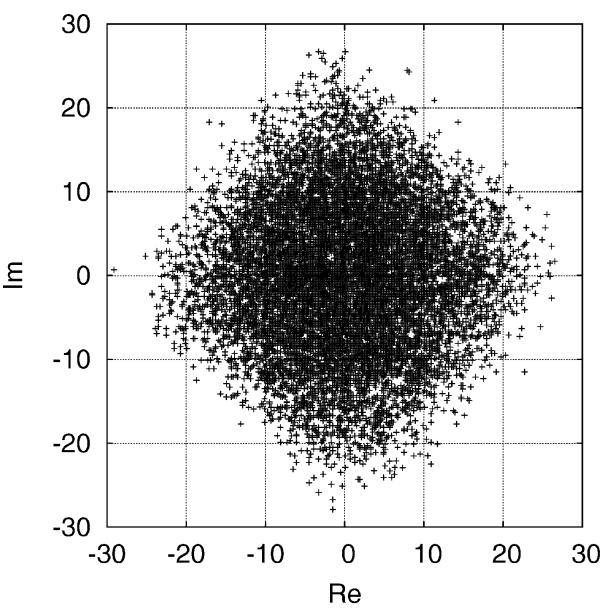

(a)

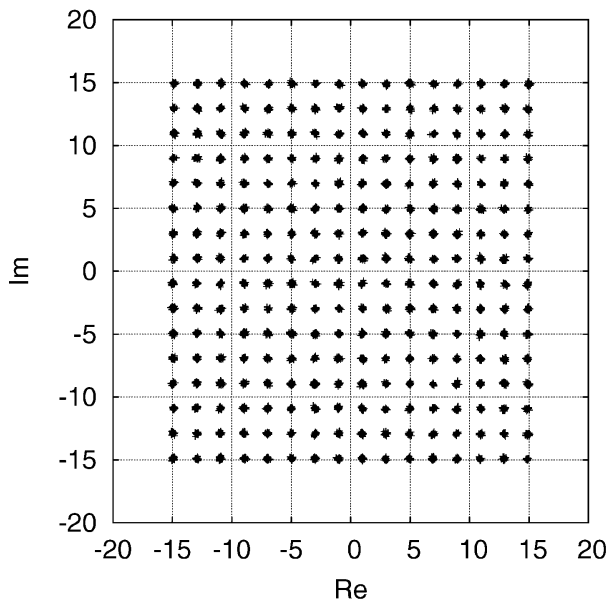

(c)

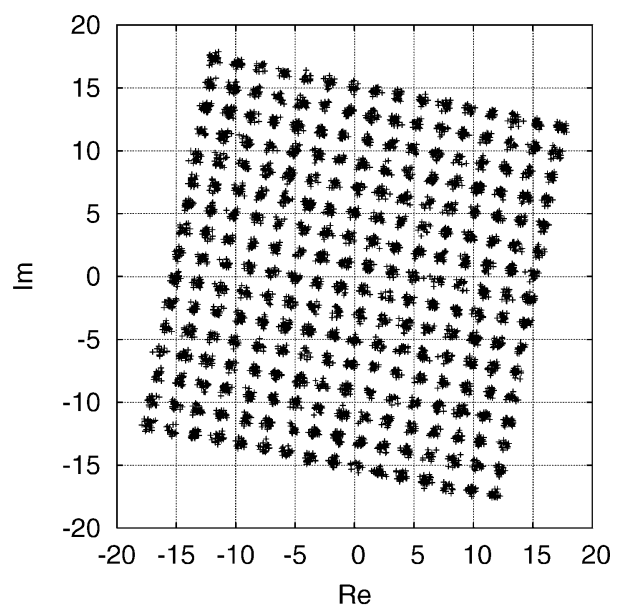

(b)

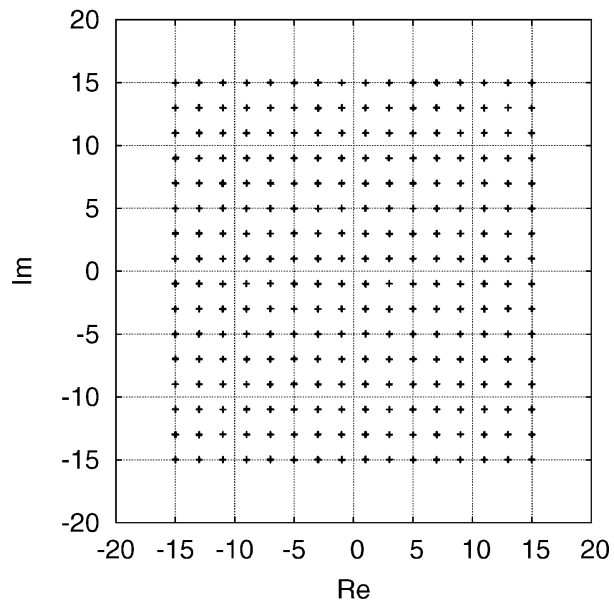

(d)

Fig. 8. Equalizer $T_{S} / 2$-spaced input (a), and equalizer $T_{S}$-spaced output signal constellations after convergence (b) the CMA, (c) the CMA+DD, and (d) bootstrap MAP for Example 3.

CMA+DD equalizer were set to $\mu_{c}=10^{-8}$ and $\mu_{d}=0.00001$. As this was a 256-QAM case, a four-stage process was adopted by the bootstrap MAP equalizer. In the first stage, $2000 T_{s}$-spaced samples were used with $\mu=0.00005$ and $\rho=30.0$. For the second stage, 2000 samples were used with $\mu=0.00005$ and $\rho=8.0$. In the third stage, 2000 samples were used with $\mu=0.00005$ and $\rho=1.6$. In the final fourth stage, the adaptive gain and cluster width were set to $\mu=0.00005$ and $\rho=0.6$, respectively. 
The learning curves of the three blind equalizers, in terms of the estimated MSE and MD measure, are depicted in Figs. $7 \mathrm{a}$ and $7 \mathrm{~b}$, respectively. The equalizer output signal constellations of the three equalizers after convergence are plotted in Fig. 8. The results again show that both the concurrent CMA+DD and bootstrap MAP equalizers have significantly better equalization performance over the CMA. It can also be seen that for this example the bootstrap MAP equalizer converges faster with better performance than the concurrent CMA+DD equalizer. In fact, the signal constellation of the bootstrap MAP equalizer depicted in Fig. 8d shows that a (near) perfect reconstruction of the transmitted signal constellation is achieved.

\section{Example 4}

For this example, 256-QAM data symbols were transmitted through a $T_{S} / 2$-spaced 22-tap channel whose CIR is given in Table 3. The noise power was set to $\sigma_{e}^{2}=4.24 \times$ $10^{-5}$, corresponding to a SNR of $60 \mathrm{~dB}$. The $T_{S} / 2$-spaced equalizer had 26 taps and the length of the data block for estimating the MSE at each adaptation was $N_{\text {MSE }}=1000$. Although this $T_{S} / 2$-spaced CIR appears to be very long, its leading and tailing taps are very small, and the effective CIR length is much smaller than 22 . We found out that a $T_{s} / 2$-spaced equalizer with 26 taps was sufficient to achieve a perfect reconstruction in the noise-free case. The adaptive gain for the CMA had to be set to $\mu=10^{-8}$ to avoid divergence. The two adaptive gains of the concurrent CMA+DD equalizer were set to $\mu_{c}=10^{-8}$ and $\mu_{d}=0.00001$. In the first three stages of the bootstrap MAP equalizer, $2000 T_{s}$-spaced samples were used in each stage with $\mu=0.00002$ and $\rho=30.0$ for stage one, $\mu=0.00002$ and $\rho=8.0$ for stage two, and $\mu=0.00002$ and $\rho=1.6$ for stage three. In the final fourth stage, the adaptive gain and cluster width were set to $\mu=0.00005$ and $\rho=0.6$, respectively.

The learning curves of the three blind equalizers, in terms of the estimated MSE and MD measure, are depicted in Figs. 9a and 9b, respectively. The equalizer output signal constellations of the three equalizers after convergence are plotted in Fig. 10. The results again show that for this example the bootstrap MAP equalizer converges faster and has better equalization performance than the concurrent CMA+DD equalizer. The signal con-

Table 3

A simulated $T_{S} / 2$-spaced 22-tap channel impulse response, where $T_{S}$ denotes symbol period

\begin{tabular}{|c|c|c|c|c|c|}
\hline Tap no. & Real & Imaginary & Tap no. & Real & Imaginary \\
\hline 0 & 0.0145 & -0.0006 & 11 & 0.0294 & -0.0049 \\
\hline 1 & 0.0750 & 0.0176 & 12 & -0.0181 & 0.0032 \\
\hline 2 & 0.3951 & 0.0033 & 13 & 0.0091 & 0.0003 \\
\hline 3 & 0.7491 & -0.1718 & 14 & -0.0038 & -0.0023 \\
\hline 4 & 0.1951 & 0.0972 & 15 & 0.0019 & 0.0027 \\
\hline 5 & -0.2856 & 0.1896 & 16 & -0.0018 & -0.0014 \\
\hline 6 & 0.0575 & -0.2096 & 17 & 0.0006 & 0.0003 \\
\hline 7 & 0.0655 & 0.1139 & 18 & 0.0005 & 0.0000 \\
\hline 8 & -0.0825 & -0.0424 & 19 & -0.0008 & -0.0001 \\
\hline 9 & 0.0623 & 0.0085 & 20 & 0.0000 & -0.0002 \\
\hline 10 & -0.0438 & 0.0034 & 21 & 0.0001 & 0.0006 \\
\hline
\end{tabular}




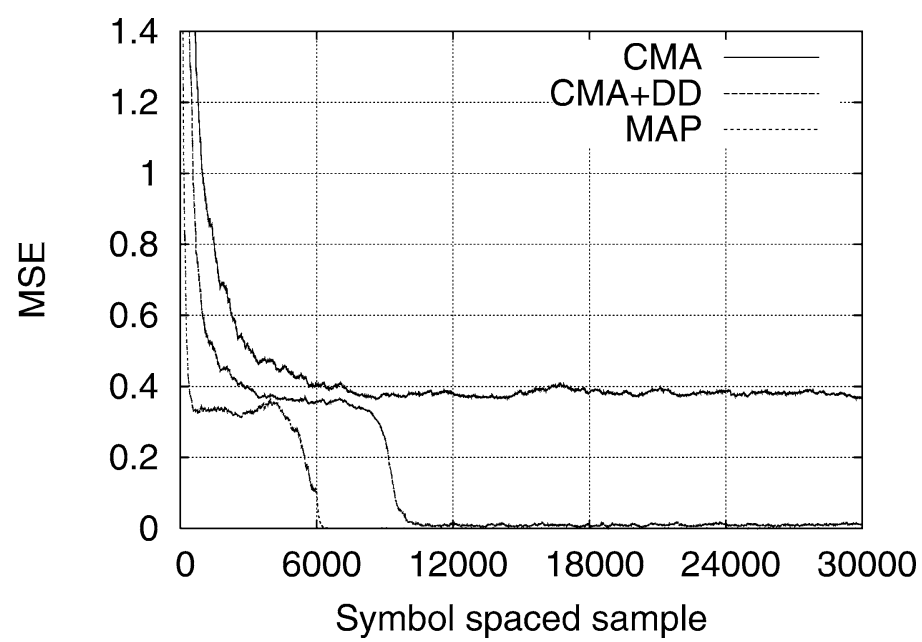

(a)

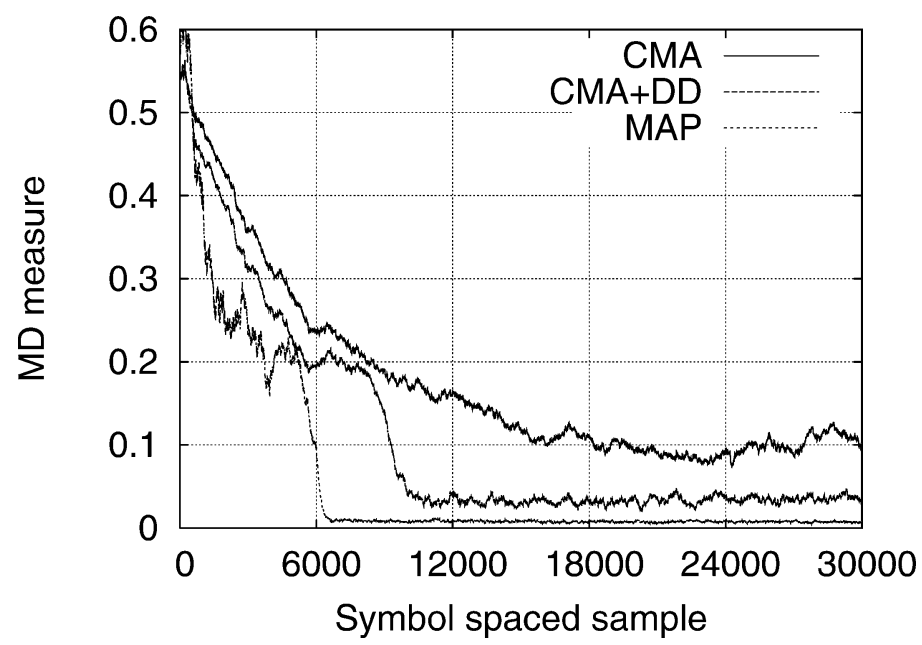

(b)

Fig. 9. Comparison of convergence performance in terms of (a) estimated MSE and (b) MD measure for Example 4.

stellation of the bootstrap MAP equalizer depicted in Fig. 10d indicates that a perfect reconstruction of the transmitted signal constellation is achieved.

\section{Conclusions}

In this paper, we have investigated two novel blind FIR equalizers, namely the concurrent CMA+DD and bootstrap MAP, with the popular CMA as a benchmark, under the condition of carrier lock and constellation registration. These two novel blind FIR equaliz- 


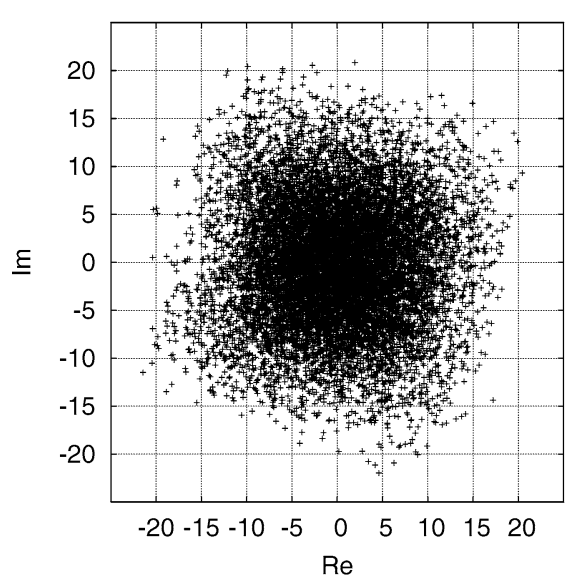

(a)

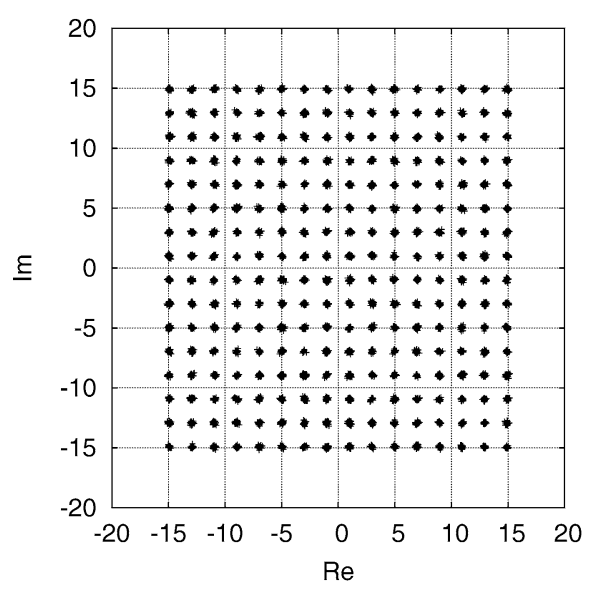

(c)

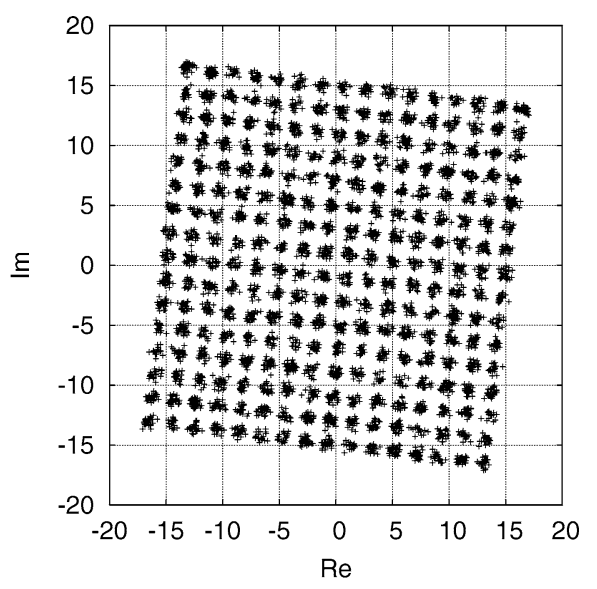

(b)

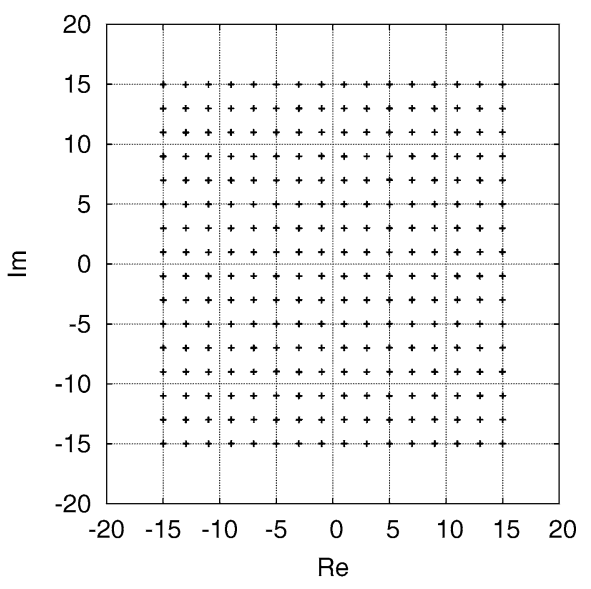

(d)

Fig. 10. Equalizer $T_{S} / 2$-spaced input (a), and equalizer $T_{S}$-spaced output signal constellations after convergence (b) the CMA, (c) the CMA+DD, and (d) bootstrap MAP for Example 4.

ers are attractive as they have low computational requirements that are only slightly more complex than the very simple CMA. Simulation study has confirmed that these two blind equalizers outperform the CMA considerably. The results have also demonstrated that the bootstrap MAP equalizer has a faster convergence speed and better steady-state performance than the concurrent CMA+DD equalizer. The tuning of the bootstrap MAP equalizer is however more complicated than that of the concurrent CMA+DD equalizer. This initial investigation suggests that further theoretical study of the concurrent CMA+DD and bootstrap MAP blind FIR equalizers is warranted. 


\section{References}

[1] D. Godard, Self-recovering equalization and carrier tracking in two-dimensional data communication systems, IEEE Trans. Commun. COM-28 (1980) 1867-1875.

[2] J.R. Treichler, B.G. Agee, A new approach to multipath correction of constant modulus signals, IEEE Trans. Acoustics Speech Signal Process. ASSP-31 (1983) 459-472.

[3] J.R. Treichler, Application of blind equalization techniques to voiceband and RF modems, in: Preprints 4th IFAC International Symposium Adaptive Systems in Control and Signal Processing, France, 1992, pp. 705713.

[4] N.K. Jablon, Joint blind equalization, carrier recovery, and timing recovery for high-order QAM signal constellations, IEEE Trans. Signal Process. 40 (1992) 1383-1389.

[5] O. Macchi, E. Eweda, Convergence analysis of self-adaptive equalizers, IEEE Trans. Inform. Theory IT-30 (1984) 161-176.

[6] F.C.C. De Castro, M.C.F. De Castro, D.S. Arantes, Concurrent blind deconvolution for channel equalization, in: Proc. ICC'2001, Vol. 2, Helsinki, Finland, June, 2001, pp. 366-371.

[7] A. Benveniste, M. Goursat, Blind equalizers, IEEE Trans. Commun. COM-32 (1984) 871-883.

[8] S. Bellini, F. Rocca, Blind deconvolution: polyspectra or Bussgang techniques?, in: E. Biglieri, G. Prati (Eds.), Digital Communications, North-Holland, Amsterdam, 1986, pp. 251-263.

[9] G. Picchi, G. Prati, Blind equalization and carrier recovering using a "stop-and-go" decision directed algorithm, IEEE Trans. Commun. COM-35 (1987) 877-887.

[10] S. Haykin (Ed.), Blind Deconvolution, Prentice Hall, Englewood Cliffs, NJ, 1994.

[11] S. Chen, S. McLaughlin, P.M. Grant, B. Mulgrew, Reduced-complexity multi-stage blind clustering equaliser, in: Proc. ICC '93, Vol. 2, Geneva, Switzerland, 1993, pp. 1149-1153.

[12] S. Chen, S. McLaughlin, P.M. Grant, B. Mulgrew, Multi-stage blind clustering equaliser, IEEE Trans. Commun. 43 (1995) 701-705.

[13] J. Karaoguz, S.H. Ardalan, A soft decision-directed blind equalization algorithm applied to equalization of mobile communication channels, in: Proc. ICC '92, Vol. 3, Chicago, USA, 1992, pp. 343.4.1-343.4.5.

[14] R. Johnson Jr., P. Schniter, T.J. Endres, J.D. Behm, D.R. Brown, R.A. Casas, Blind equalization using the constant modulus criterion: a review, Proc. IEEE 86 (1998) 1927-1950. 